\section{Development of an Authentication System for Genuine Radix Salviae Miltiorrhizae (Salvia miltiorrhiza) Using SNP Markers}

\section{Tiantian Zhang, Jing Wang, Di Wang, Hongtao Wang}

College of Life Science, Yantai University, Yantai, P. R. China

\section{Abstract \\ $\nabla$}

Radix Salviae Miltiorrhizae (Salvia miltiorrhiza) is one of the most widely used traditional Chinese medicinal materials. However, the dried roots of Salvia przewalskii, Salvia yunnanensis, Salvia sinica, and Salvia digitaloides are usually mistaken for Radix Salviae Miltiorrhizae due to their similarity in appearance. Although several efforts have been made to develop molecular markers for Salvia species, the discrimination of Radix Salviae Miltiorrhizae from its adulterants is still an unresolved problem. In order to develop a simple and reliable method for identifying genuine Radix Salviae Miltiorrhizae, the nuclear internal transcribed spacer region was analyzed to exploit DNA polymorphisms, and a host of single nucleotide polymorphism sites were discovered among five Salvia species. By introducing additional mismatches, allelespecific primers were designed and a multiplex polymerase chain reaction was conducted for authentication of Radix Salviae Miltiorrhizae. The established multiplex allele-specific polymerase chain reaction system enabled the simultaneous identification of four Salvia species in one reaction. Therefore, a simple and reliable method for molecular authentication of S. miltiorrhi$z a$ from its adulterants was established, and the present method should be an important tool to complement morphological and chemical analysis for quality control of Radix Salviae Miltiorrhizae.

\section{Abbreviations \\ $\nabla$ \\ ITS: $\quad$ internal transcribed spacer \\ SNP: $\quad$ single nucleotide polymorphism \\ RAPD: $\quad$ random amplified polymorphic DNA \\ ISSR: inter-simple sequence repeats \\ RFLP: $\quad$ restriction fragment length polymorphism \\ AFLP: amplified fragments length polymorphism \\ SRAP: $\quad$ sequence related amplified polymorphism \\ CoRAP: conserved region amplification polymorphism}

Key words

Salvia miltiorrhiza - Lamiaceae - Radix Salviae Miltiorrhizae • ITS $\cdot$ SNP $\cdot$ allele-specific PCR

Salvia miltiorrhiza Bunge (Lamiaceae) is one of the most widely used traditional Chinese medicinal plants. The dried root and rhizome of S. miltiorrhiza, known as Radix Salviae Miltiorrhizae (Danshen in Chinese), have been used for the treatment of various cardiovascular and cerebrovascular diseases for hundreds of years in Asian countries [1]. Modern pharmacologic studies have demonstrated that Radix Salviae Miltiorrhizae has additional biological activities including anticancer, anti-inflammatory, antioxidant, antimicrobial, and antivirus [2]. Due to its comprehen- sive and remarkable pharmacological activities, especially its antioxidant capacity, Radix Salviae Miltiorrhizae has become a widely accepted health-promoting product and worldwide consumption is rapidly increasing [3].

A common problem existing in traditional Chinese medicine materials is that substitutes and adulterants are often introduced intentionally or accidentally, and Radix Salviae Miltiorrhizae is no exception. In the Salvia genus, only S. miltiorrhiza is regarded as the botanical source of Radix Salviae Miltiorrhizae in the Pharmacopoeia of People's Republic of China [4]. However, the dried roots of Salvia przewalskii Maxim., Salvia yunnanensis C.H. Wright, Salvia sinica Migo, and Salvia digitaloides Diels are usually mistaken for Radix Salviae Miltiorrhizae in some local areas in China [5]. Therefore, a simple and reliable method is clearly needed for the accurate identification of Radix Salviae Miltiorrhizae in order to ensure its clinical efficacy and safety as well as to protect consumer's rights.

Traditional methods based on morphological characteristics for authentication of Radix Salviae Miltiorrhizae are subjective and error-prone, as Radix Salviae Miltiorrhizae and its adulterants resemble each other in shape and color [6]. Chemical constituents such as tabshinone and cryptotanshinone are used as identification markers as well [7], but their contents may be affected by physiological conditions and many Salvia species contain similar chemical components [8]. With the development of molecular biology techniques, DNA markers have become a popular means for authentication of Radix Salviae Miltiorrhizae. Although several efforts have been made to develop molecular markers for Salvia species, the discrimination of Radix Salviae Miltiorrhizae from its adulterants is still an unresolved problem. In this study, we present a simple and reliable method for identifying genuine Radix Salviae Miltiorrhizae from its adulterants by using SNP markers and multiplex allele-specific PCR.

Proven to be an ideal DNA barcode for species identification [9, 10], sequences of the nuclear ribosomal ITS region have been evaluated for genotyping Salvia species $[7,11,12]$. But specific authentication of $S$. mitiorrhiza from its adulterants was scarcely studied, especially the use of many Salvia species going under the name of Radix Salviae Miltiorrhizae. Therefore, we tried to establish a simple and reliable DNA method for Radix Salviae Miltiorrhizae authentication.

The compiled ITS sequences of five Salvia species were registered in GenBank with accession numbers of KJ397256-KJ397260. From the multiple sequence alignment result, as shown in - Fig. 1, a host of SNP sites exist in the ITS region among five Salvia species. However, an SNP site specific for S. mitiorrhiza was discovered neither in the ITS1 region nor in the ITS2 region among five species. Accordingly, the authentication of Radix Salviae Miltiorrhizae was conducted using the combination of mutation sites common to two or three species via multiplex allelespecific PCR.

At the nucleotide positions of 75 bp and 76 bp in ITS1, S. miltiorrhiza, S. sinica, and S. yunnanensis contain nucleotide A and T, while $S$. przewalskii and S. digitaloides were replaced with $\mathrm{T}$ and $\mathrm{C}$ in the same positions. These two mutation sites were chosen to design primer SMR for the former three species mentioned above. Primer SYR was designed for both S. miltiorrhiza and S. yunnanensis based on the SNP site at the nucleotide position of $538 \mathrm{bp}$, with an additional mismatch of A for T. Similarly, primer SSR was designed for specific authentication of $S$. sinica, with a substitution of $\mathrm{G}$ for $\mathrm{T}$, to ensure reliable discrimination ( $\bullet$ Table 


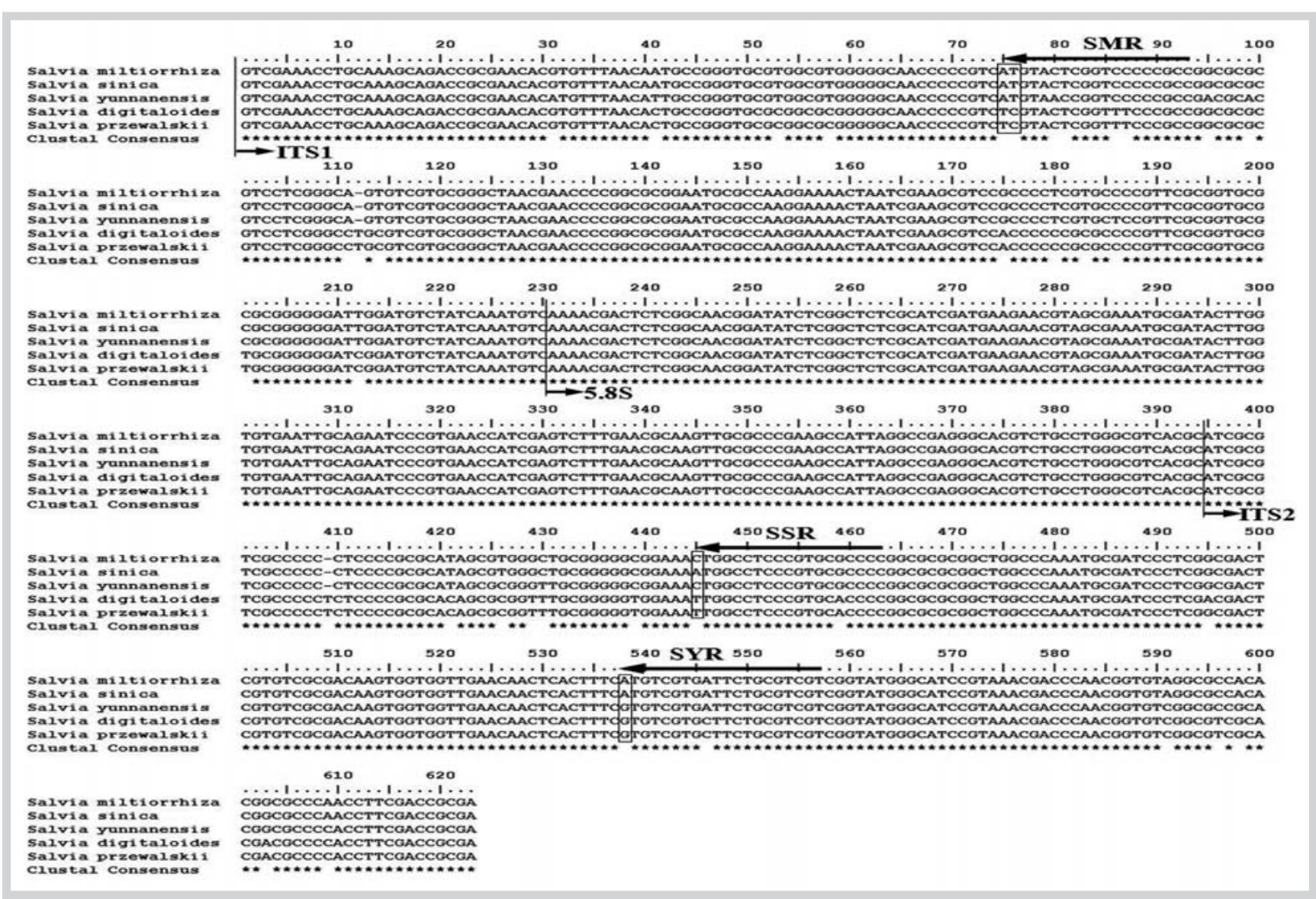

Fig. 1 Comparison of ITS sequences of five Salvia species.

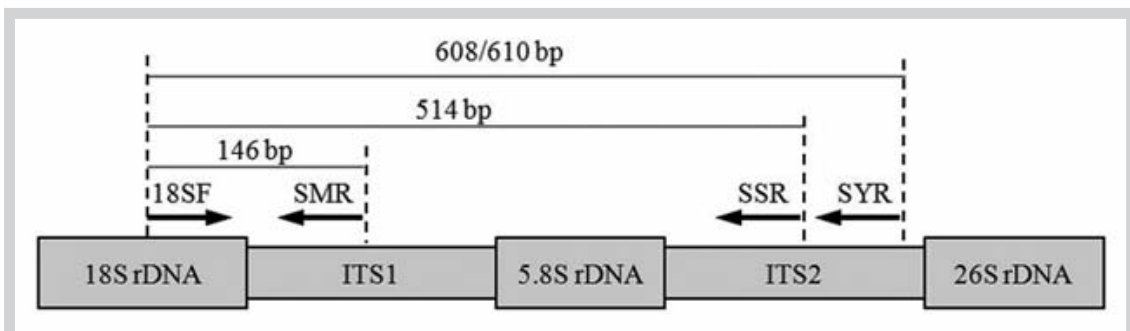

Fig. 2 Positions of primers used in this study.

1). Additionally, dimmers, hairpins, and false primings are avoided as far as possible to minimize false positive results and ensure amplification efficiency [13].

With the combination of these three primers, as well as the forward primer 18SF, multiplex allele-specific PCR was performed to identify $S$. miltiorrhiza from its adulterants. As expected, the combination of three primer pairs generated different fragment patterns for different Salvia species (๑ Fig. 3). S. miltiorrhiza, S. sinica, and S. yunnanensis yielded their specific amplicons of 146 bp, which represents their AT allele. Likewise, only S. yunnanensis, S. przewalskii, and S. digitaloides could be amplified by primer SYR, with their bands of 608 bp, 610 bp, and 610 bp, respectively. The fragments of $514 \mathrm{bp}$, from which $\mathrm{S}$. sinica can be differentiated among the other four species, were produced by primer SSR, representing its specific A allele. From the fragment patterns, $S$. miltiorrhiza can be easily discriminated from its four adulterants by its single $146 \mathrm{bp}$ fragment, which differs from the 610 bp specific bands of $S$. przewalskii and S. digitaloides.
Table 1 Primers used in this study.

\begin{tabular}{lll}
$\begin{array}{l}\text { Primer } \\
\text { name }\end{array}$ & $\begin{array}{l}\text { Nucleotide sequence }\left(\mathbf{5}^{\prime} \rightarrow \mathbf{3}^{\prime}\right) \\
\text { 18SF }\end{array}$ & $\begin{array}{l}\text { Position in } \\
\text { ITS }\end{array}$ \\
\hline 26SR & GGAAGTAAAAGTCGTAACAAGG & \\
\hline SMR & TCCTCCGCTTATTGATATGC & \\
SSR & GGCGGGGGACCGAGTACAT & $93-75$ \\
\hline SYR & GGGTCGCACGGGAGGCTAT $(G \rightarrow T)$ & $463-445$ \\
\hline
\end{tabular}

Bold nucleotides are additional mismatches introduced intentionally

Although S. sinica and S. yunnanensis also generated the $146 \mathrm{bp}$ fragment, they can be clearly identified by their specific amplicons of 514 bp and 608 bp, respectively. Therefore, the established multiplex allele-specific PCR system is effective for authentication of $S$. miltiorrhiza from its adulterants. 


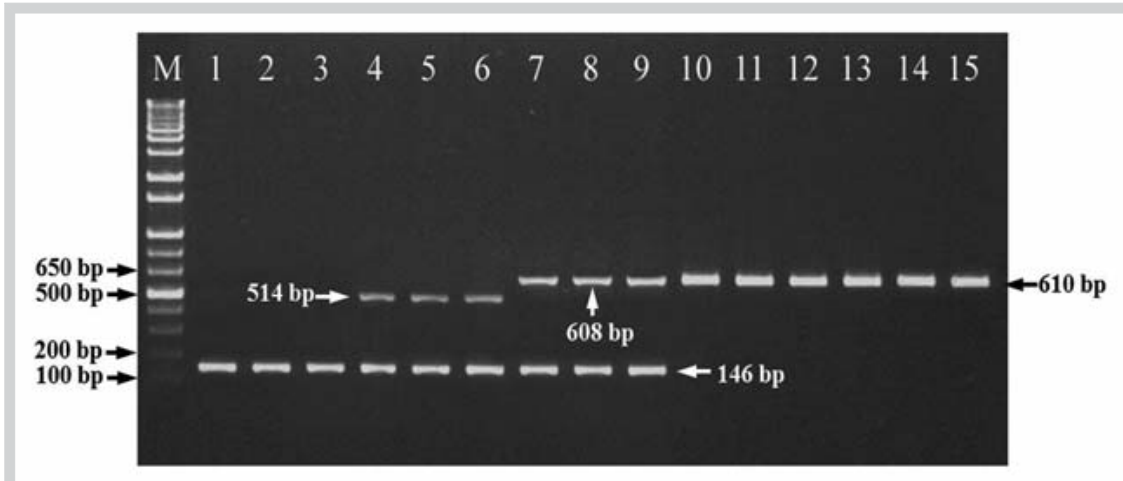

Fig. 3 Multiplex PCR products of five Salvia species. Lane M: 1 kb DNA ladder; lanes 1-3: S. mitiorrhiza; lanes 4-6: S. sinica; lanes 7-9: S. yunnanensis; lanes 10-12: S. digitaloides; lanes 13-15: S. przewalskii.

\begin{tabular}{|c|c|c|c|}
\hline Species & Locations & Voucher specimen & Genbank accession no. \\
\hline S. miltiorrhiza & Shandong, China & SM201301 & \multirow[t]{3}{*}{ KJ397256 } \\
\hline S. miltiorrhiza & Henan, China & SM201302 & \\
\hline S. miltiorrhiza & Sichuan, China & SM201303 & \\
\hline S. sinica & Anhui, China & SS201301 & \multirow[t]{3}{*}{ KJ397257 } \\
\hline S. sinica & Zhejiang, China & SS201302 & \\
\hline S. sinica & Hubei, China & SS201303 & \\
\hline S. yunnanensis & Yunnan, China & SY201301 & \multirow[t]{3}{*}{ KJ397258 } \\
\hline S. yunnanensis & Yunnan, China & SY201302 & \\
\hline S. yunnanensis & Sichuan, China & SY201303 & \\
\hline S. digitaloides & Yunnan, China & SD201301 & \multirow[t]{3}{*}{ KJ397259 } \\
\hline S. digitaloides & Yunnan, China & SD201302 & \\
\hline S. digitaloides & Yunnan, China & SD201303 & \\
\hline S. przewalskii & Sichuan, China & SP201301 & \multirow[t]{3}{*}{ KJ397260 } \\
\hline S. przewalskii & Yunnan, China & SP201302 & \\
\hline S. przewalskii & Yunnan, China & SP201303 & \\
\hline
\end{tabular}

Table 2 Plant samples used in this study.
Due to the potential confusion over which variety of the medicinal plant is called for in a given situation, accurate authentication is critical for its effective and safe application. This is particularly true in the context of the Salvia genus in which many species are usually mistaken for Radix Salviae Miltiorrhizae in some local areas in China. Nowadays, molecular technology provides an independent approach for the authentication of medicinal plants. Several DNA molecular markers have been developed for Salvia species, including RAPD [14], AFLP [15], PCR-RFLP [16], SRAP [17], CoRAP [18], and ISSR [19]. However, these methods are not suitable for developing a simple and reliable method to identify Radix Salviae Miltiorrhizae,mainly due to their sensitivity to PCR temperature or tedious visualization procedures.

Although SNP site specific for S. miltiorrhiza does not exist in the ITS region, molecular authentication of Radix Salviae Miltiorrhizae from its adulterants was achieved by SNP genotyping with a multiplex allele-specific PCR. The introduction of additional mismatches ensured a reliable discrimination between alleles and multiplex PCR-enabled identification of Radix Salviae Miltiorrhizae. Besides, the established multiplex allele-specific PCR system enables the simultaneous identification of four Salvia species in one reaction, requires no sequencing analysis of PCR products, and the authentication of Radix Salviae Miltiorrhizae only needs a simple agarose gel-based assay after PCR. Compared with the developed DNA markers for Salvia species, the present method is simple, reliable, and cost-effective. The ITS region appears in multiple copies in the nuclear genome, and PCR amplification is thus not appreciably affected by DNA degradation. Therefore, this method is strongly recommended for the authentication of pro- cessed Radix Salviae Miltiorrhizae materials. In this study, a simple and reliable method for molecular authentication of S. miltiorrhiza from its adulterants was established by SNP genotyping and multiplex PCR. Because chemical compositions are usually affected by environmental and processing factors, the present method should be an important tool to complement morphological and chemical analyses for quality control of Radix Salviae Miltiorrhizae.

\section{Materials and Methods \\ $\nabla$}

Plant materials and DNA isolation

The plant samples listed in $\bullet$ Table 2 were collected from different regions of China and identified by Prof. Haizhu Jin, College of Life Science, Yantai University. All the voucher specimens were deposited in the Research Institute of Food Science and Technology, Yantai University. The plant leaves and medicinal materials were frozen in liquid nitrogen and ground into a fine powder. Genomic DNA was then isolated and purified using a plant DNA isolation kit (Exgene Tissue SV, GeneAll), according to the manufacturer's instructions.

\section{Polymerase chain reaction amplification of} internal transcribed spacer region and multiple sequence alignment

The ribosomal ITS region was amplified using forward primer 18SF (5'-GGAAGTAAAAGTCGTAACAAGG-3') and reverse primer 26SR (5'-TCCTCCGCTTATTGATATGC-3'). The $20 \mu \mathrm{L}$ PCR reaction mixture contained $20 \mathrm{ng}$ of template DNA, $0.5 \mu \mathrm{M}$ of each primer, 
and $10 \mu \mathrm{L}$ of $2 \mathrm{X}$ PreMix DNA polymerase (Genotech). The PCR amplification profile consisted of 1 predenaturation cycle of $4 \mathrm{~min}$ at $94^{\circ} \mathrm{C}$, followed by 35 cycles of denaturation at $94^{\circ} \mathrm{C}$ for $30 \mathrm{~s}$, annealing at $58^{\circ} \mathrm{C}$ for $30 \mathrm{~s}$, extension at $72^{\circ} \mathrm{C}$ for $30 \mathrm{~s}$, and a final extension at $72^{\circ} \mathrm{C}$ for $5 \mathrm{~min}$. PCR products were analyzed by electrophoresis on $1.0 \%$ agarose gel and then purified with a PCR DNA Purification Kit (GeneAll) as described in the manufacturer's instructions. DNA was sequenced in both directions on an automatic DNA sequencer (ABIPRISM 3700) by using a BigDye Terminator Cycle Sequencing Kit (Applied Biosystems). DNA sequences were assembled using SeqMan software, and multiple sequence alignments were conducted using the ClustalW2.0 program [20].

\section{Design of specific primers}

Primers were designed based on the SNP sites detected from the multiple alignment result. The substitutions of $\mathrm{G}$ for $\mathrm{T}$ in primer SSR and A for T in primer SYR were introduced deliberately to ensure absolute specificity and reliable discrimination between alleles [21]. Primer SMR was also designed to perform multiplex PCR with SSR and SYR for Radix Salviae Miltiorrhizae authentication. The locations of primers used in this study are shown in - Fig. 2.

\section{Multiplex allele-specific polymerase chain reaction}

Three new designed primers, together with the ITS forward primer 18SF, were used for authentication of Radix Salviae Miltiorrhizae by multiplex PCR. The $20 \mu \mathrm{L}$ PCR reaction mixture consisted of $50 \mathrm{ng}$ of DNA template and $10 \mu \mathrm{L}$ of $2 \mathrm{X}$ Premix DNA polymerase (Genotech). The concentrations of four primers, 18SF, SMR, SSR, and SYR, were $0.5 \mu \mathrm{M}, 0.125 \mu \mathrm{M}, 0.3 \mu \mathrm{M}$, and $0.5 \mu \mathrm{M}$, respectively. Multiplex PCR was performed using 1 cycle of $4 \mathrm{~min}$ at $94^{\circ} \mathrm{C}, 33$ cycles of $30 \mathrm{~s}$ at $94^{\circ} \mathrm{C}, 30 \mathrm{~s}$ at $62^{\circ} \mathrm{C}$, and $30 \mathrm{~s}$ at $72^{\circ} \mathrm{C}$ with a final extension at $72^{\circ} \mathrm{C}$ for $5 \mathrm{~min}$.

\section{Acknowledgements}

$\nabla$

The present study was supported by the Shandong Provincial Natural Science Foundation (ZR2013CQ021) and the Scientific Research Foundation for the Returned Overseas Chinese Scholars, State Education Ministry.

\section{Conflict of Interest}

$\nabla$

The authors declare of no conflict of interest.

\section{References}

1 Wang X, Morris-Natschke SL, Lee KH. New developments in the chemistry and biology of the bioactive constituents of Tanshen. Med Res Rev 2007; 27: 133-148

2 Zhou L, Zuo Z, Chow MS. Danshen: an overview of its chemistry, pharmacology, pharmacokinetics, and clinical use. J Clin Pharmacol 2005; 45: 1345-1359

3 Li YG, Song L, Liu M, Hu ZB, Wang ZT. Advancement in analysis of Salviae miltiorrhizae Radix et Rhizoma (Danshen). J Chromatogr A 2009; 1216: 1941-1953

4 Chinese Pharmacopoeia Commission. Pharmacopoeia of the People's Republic of China. Beijing: People's Medical Publishing House; 2010: 70

5 National Institutes for Food and Drug Control and Guangdong Institute for Drug control. Identification of Pictorial Handbook of Chinese Medic- inal Materials (Book 2). Guangzhou: Guangdong Science \& Technology Press; 2011: 49-50

6 Korir NK, Han J, Shangguan L, Wang C, Kayesh E, Zhang Y, Fang J. Plant variety and cultivar identification: advances and prospects. Crit Rev Biotechnol 2013; 33: 111-125

$7 \mathrm{Xu} H$, Wang ZT, Cheng KT, Wu T, Gu LH, Hu ZB. Comparison of rDNA ITS sequences and tanshinones between Salvia miltiorrhiza populations and Salvia species. Bot Stud 2009; 50: 127-135

8 Zhong GX, Li P, Zeng LJ, Guan J, Li DQ Li SP. Chemical characteristics of Salvia miltiorrhiza (Danshen) collected from different locations in China. J Agric Food Chem 2009; 57: 6879-6887

9 Yao H, Song J, Liu C, Luo K, Han J, Li Y, Pang X, Xu H, Zhu Y, Xiao P. Use of ITS2 region as the universal DNA barcode for plants and animals. PLoS One 2010; 5: e13102

10 Hollingsworth PM, Graham SW, Little DP. Choosing and using a plant DNA barcode. PLoS One 2011; 6: e19254

11 Han JP, Liu C, Shi LC, Chen XC, Lim YL. Comparison of four DNA barcodes in identifying certain medicinal plants of Lamiaceae. J Syst Evol 2012; 50: 227-234

12 Wang M, Zhao HX, Wang L, Wang T, Yang RW, Wang XL, Zhou YH, Ding CB, Zhang $L$. Potential use of DNA barcoding for the identification of Salvia based on cpDNA and nrDNA sequences. Gene 2013; 528: 206-215

13 Wang H, Sun H, Kwon WS, Jin H, Yang DC. A simplified method for identifying the Panax ginseng cultivar Gumpoong based on $26 \mathrm{~S}$ rDNA. Planta Med 2010; 76: 399-401

14 Wang M, Li J, Zhang L, Yang R, Ding C, Zhou Y, Yin Z. Genetic diversity among Salvia miltiorrhiza Bunge and related species using morphological traits and RAPD markers. J Med Plant Res 2011; 5: 2687-2694

15 Braglia L, Casabianca V, De Benedetti L, Pecchioni N, Mercuri A, Cervelli C, Ruffoni B. Amplified fragment length polymorphism markers for DNA fingerprinting in the genus Salvia. Plant Biosyst 2011; 145: 274-277

16 Karaca M, Ince AG, Ay ST, Turgut K, Onus AN. PCR-RFLP and DAMD-PCR genotyping for Salvia species. J Sci Food Agric 2008; 88: 2508-2516

17 Song Z, Li X, Wang H, Wang J. Genetic diversity and population structure of Salvia miltiorrhiza Bge in China revealed by ISSR and SRAP. Genetica 2010; 138: 241-249

18 Wang $Q$ Z Zhang B, Lu Q. Conserved region amplification polymorphism (CoRAP), a novel marker technique for plant genotyping in Salvia miltiorrhiza. Plant Mol Biol Rep 2009; 27: 139-143

19 Zhang Y, Li X, Wang Z. Diversity evaluation of Salvia miltiorrhiza using ISSR markers. Biochem Genet 2013; 51: 707-721

20 Larkin M, Blackshields G, Brown N, Chenna R, McGettigan PA, McWilliam $H$, Valentin F, Wallace IM, Wilm A, Lopez R. Clustal W and Clustal X version 2.0. Bioinformatics 2007; 23: 2947-2948

21 Drenkard E, Richter BG, Rozen S, Stutius LM, Angell NA, Mindrinos M, Cho RJ, Oefner PJ, Davis RW, Ausubel FM. A simple procedure for the analysis of single nucleotide polymorphisms facilitates map-based cloning in Arabidopsis. Plant Physiol 2000; 124: 1483-1492

received August 14, 2015

revised September 27, 2015

accepted September 30, 2015

Bibliography

DOI http://dx.doi.org/10.1055/s-0035-1558205

Planta Med Lett 2015; 2: e65-e68

(c) Georg Thieme Verlag KG Stuttgart · New York . ISSN 2199-157X

\section{Correspondence}

\section{Hongtao Wang, PhD, Associate Professor}

College of Life Science

Yantai University

NO. 30 Qingquan street

Yantai, 264005

P.R. China

Phone: + 865356913938

Fax: +865356902638

ytuwht@163.com 